\title{
Aerosol retrieval from multiangle, multispectral photopolarimetric measurements: importance of spectral range and angular resolution
}

\author{
L. Wu ${ }^{1,4}$, O. Hasekamp ${ }^{1}$, B. van Diedenhoven ${ }^{2,3}$, and B. Cairns ${ }^{3}$ \\ ${ }^{1}$ SRON Netherlands Institute for Space Research, Sorbonnelaan 2, 3584 CA Utrecht, \\ the Netherlands \\ ${ }^{2}$ Columbia University, Center for Climate System Research, New York, NY, USA \\ ${ }^{3}$ NASA Goddard Institute for Space Studies, New York, NY, USA \\ ${ }^{4}$ School of Computer and Information, Hefei University of Technology, Hefei, \\ Anhui 230009, China \\ Correspondence to: $\mathrm{L} . \mathrm{Wu}(\mathrm{l} . w \mathrm{wu}$ sron.nl)
}

Received: 18 February 2015 - Published in Atmos. Meas. Tech. Discuss.: 13 March 2015

Revised: 05 June 2015 - Accepted: 08 June 2015 - Published: 29 June 2015

\begin{abstract}
We investigated the importance of spectral range and angular resolution for aerosol retrieval from multiangle photopolarimetric measurements over land. For this purpose, we use an extensive set of simulated measurements for different spectral ranges and angular resolutions and subsets of real measurements of the airborne Research Scanning Polarimeter (RSP) carried out during the PODEX and SEAC ${ }^{4} \mathrm{RS}$ campaigns over the continental USA. Aerosol retrievals performed from RSP measurements show good agreement with ground-based AERONET measurements for aerosol optical depth (AOD), single scattering albedo (SSA) and refractive index. Furthermore, we found that inclusion of shortwave infrared bands (1590 and/or $2250 \mathrm{~nm}$ ) significantly improves the retrieval of AOD, SSA and coarse mode microphysical properties. However, accuracies of the retrieved aerosol properties do not improve significantly when more than five viewing angles are used in the retrieval.
\end{abstract}

\section{Introduction}

The radiative impact caused by aerosols is widely considered to be one of the largest uncertainties in the radiative forcing of the Earth climate (IPCC, 2013). Aerosols influence the radiative balance of the Earth by scattering and absorbing solar radiation. A major issue for the evaluation of aerosols in climate models is the limited accuracy and detail of global measurements of the aerosol properties required for climate modeling, such as particle size distribution, shape, complex refractive index and columnar concentration. Orbital remote sensing instruments that perform multiangle, multispectral photopolarimetric measurements have the capability to provide these aerosol properties with the required accuracy (Mishchenko and Travis, 1997; Hasekamp and Landgraf, 2007). The advantages of polarimetric measurements over intensity-only measurements come from the sensitivity of polarization of light and its spectral and angular dependence to particle size, shape and refractive index. The Polarization and Directionality of the Earth's Reflectance (POLDER), the most recent in-orbit polarization-measuring satellite instrument, has demonstrated the importance of polarization measurements for the retrieval of aerosol properties (Hasekamp et al., 2011; Dubovik et al., 2011). Limitations of the POLDER instrument are its inherent limited polarimetric accuracy and its relatively small spectral range. To overcome these limitations, the Aerosol Polarimetry Sensor (APS) was scheduled for launch on the Glory mission. This instrument was designed to view a scene at $\sim 250$ viewing angles in nine spectral bands ranging from 410 to $2250 \mathrm{~nm}$. Due to the tragedy of the Glory launch failure and the decommissioning of POLDER, space-borne multiangle photo polarimetric measurements are currently unavailable. However, the Research Scanning Polarimeter (RSP) that has sim- 
ilar characteristics as APS continues to perform airborne measurements in many field campaigns. The collected data will facilitate algorithm development and allow us to derive geophysically relevant aerosol and cloud properties (Waquet et al., 2009a).

RSP provides measurements of a ground scene at $\sim 152$ different viewing angles $\left( \pm 60^{\circ}\right.$ from nadir) in nine spectral bands from visible/near-infrared bands to shortwave infrared bands (410, 470, 550, 670, 865, 960, 1590, 1880 and $2250 \mathrm{~nm}$ ). The measurements provide the first three Stokes parameters, $I, Q$ and $U$, in the nine spectral channels with a radiometric uncertainty of $\sim 2.0 \%$ and a polarimetric uncertainty of $\sim 0.5 \%$ (Cairns et al., 1999). Given its large spectral range and number of viewing angles, RSP measurements are well suited to study the importance of spectral range and angular resolution for the retrieval of aerosol properties. These are two important design parameters for future polarimetric instrumentation.

In this paper we apply an extended version of the SRON aerosol retrieval algorithm (Hasekamp et al., 2011) to several measurement subsets of RSP measurements over land in order to investigate the importance of spectral range and angular resolution of multiangle photopolarimetric instrumentation for aerosol retrieval. In addition, we also study the above-mentioned aspects using an extensive set of synthetic measurements. Although studies using synthetic multiangle photopolarimetric measurements have been published before (Hasekamp and Landgraf, 2007; Hasekamp, 2010; Knobelspiesse et al., 2012; Ottaviani et al., 2013), they were all based on linear error propagation and a limited number of aerosol scenarios. Instead, here we perform a full iterative retrieval on the synthetic measurements and consider a large number of aerosol scenarios. Therefore, the synthetic study included in this paper is an extension of the synthetic studies mentioned above.

In order to exploit the full spectral range of RSP, we extend the SRON aerosol retrieval algorithm to be capable to cope with spectrally dependent refractive indices. The paper is organized as follows: Sect. 2 describes the data used in the paper, Sect. 3 describes the retrieval method, Sect. 4 shows the results based on synthetic measurements and Sect. 5 shows the results based on RSP measurements. Finally, Sect. 6 concludes the paper.

\section{Data}

The RSP data used in the paper are obtained during the PODEX (Polarimeter Definition Experiment) campaign and the SEAC ${ }^{4}$ RS (Studies of Emissions and Atmospheric Composition, Clouds and Climate Coupling by Regional Surveys) campaign in 2013 (Table 1). While the objectives for both campaigns include studies for future instrument design, SEAC ${ }^{4} \mathrm{RS}$ also seeks to improve our understanding of feedbacks of aerosols on meteorology and climate (Toon, 2013).

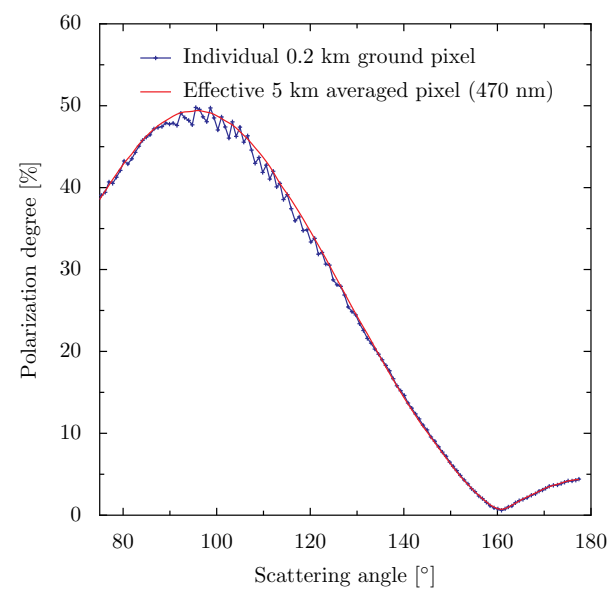

Figure 1. Measurements of individual ground pixel and effective $5 \mathrm{~km}$ averaged pixel at $470 \mathrm{~nm}$. Oscillations in angular measurements are due to imperfect co-registration of different viewing angles.

The PODEX and SEAC ${ }^{4} \mathrm{RS}$ measurements were acquired over the continental USA with RSP instrument mounted on an ER-2 high-altitude aircraft which flies at an altitude of approximately $20 \mathrm{~km}$ with a speed of around $200 \mathrm{~m} \mathrm{~s}^{-1}$. During the SEAC ${ }^{4} \mathrm{RS}$ campaign, a number of AERONET sites co-located with the ER-2 flight track are available; thus we can compare aerosol properties obtained from RSP measurements with corresponding AERONET aerosol products.

For both campaigns, we focus on measurements of the RSP bands at 410, 470, 550, 670, 865, 1590 and $2250 \mathrm{~nm}$. The 960 and $1880 \mathrm{~nm}$ bands are excluded, because their main purposes are water vapor estimation and cirrus cloud screening. For each spectral band, we only use viewing angles in the range $\left[-60,40^{\circ}\right]$ because some of the viewing angles are blocked by the ER-2 aircraft. We use the data that have already been aggregated to ground level. Due to imperfect coregistration of different viewing angles, the angular measurements show oscillations larger than the measurement error for inhomogeneous scenes. In order to reduce this effect we average RSP measurements over a distance of $5 \mathrm{~km}$ so that misregistrations between viewing angles become small compared to the effective pixel size. Figure 1 shows an example of a RSP measurement for an individual ground pixel and for an effective $5 \mathrm{~km}$ averaged pixel.

Here, information on temperature, pressure and relative humidity profiles in atmosphere is interpolated to a particular date, time and location of the RSP measurements using NCEP reanalysis data (Kistler et al., 2001). 
Table 1. Information of used RSP measurements

\begin{tabular}{|c|c|c|c|c|}
\hline Case & Campaign & Co-located AERONET site & Longitude and latitude & Date \\
\hline 1 & PODEX & Fresno_2 & $119.77^{\circ} \mathrm{W}, 36.78^{\circ} \mathrm{N}$ & 20130116 \\
\hline 2 & PODEX & Fresno_2 & $119.77^{\circ} \mathrm{W}, 36.78^{\circ} \mathrm{N}$ & 20130120 \\
\hline 3 & PODEX & DRAGON_Porterville & $119.05^{\circ} \mathrm{W}, 36.03^{\circ} \mathrm{N}$ & 20130120 \\
\hline 4 & PODEX & DRAGON_Drummond & $119.74^{\circ} \mathrm{W}, 36.70^{\circ} \mathrm{N}$ & 20130120 \\
\hline 5 & PODEX & DRAGON_Visalia & $119.39^{\circ} \mathrm{W}, 36.31^{\circ} \mathrm{N}$ & 20130122 \\
\hline 6 & $\mathrm{SEAC}^{4} \mathrm{RS}$ & Calipso_Carthage & $94.06^{\circ} \mathrm{W}, 32.06^{\circ} \mathrm{N}$ & 20130909 \\
\hline 7 & $\mathrm{SEAC}^{4} \mathrm{RS}$ & Upper_Buffalo & $93.20^{\circ} \mathrm{W}, 35.82^{\circ} \mathrm{N}$ & 20130906 \\
\hline 8 & $\mathrm{SEAC}^{4} \mathrm{RS}$ & Upper_Buffalo & $93.20^{\circ} \mathrm{W}, 35.82^{\circ} \mathrm{N}$ & 20130827 \\
\hline 9 & $\mathrm{SEAC}^{4} \mathrm{RS}$ & Leland_HS & $90.89^{\circ} \mathrm{W}, 33.40^{\circ} \mathrm{N}$ & 20130909 \\
\hline 10 & $\mathrm{SEAC}^{4} \mathrm{RS}$ & Leland_HS & $90.89^{\circ} \mathrm{W}, 33.40^{\circ} \mathrm{N}$ & 20130830 \\
\hline $11-14$ & $\mathrm{SEAC}^{4} \mathrm{RS}$ & Baskin & $91.73^{\circ} \mathrm{W}, 32.28^{\circ} \mathrm{N}$ & 20130909 \\
\hline
\end{tabular}
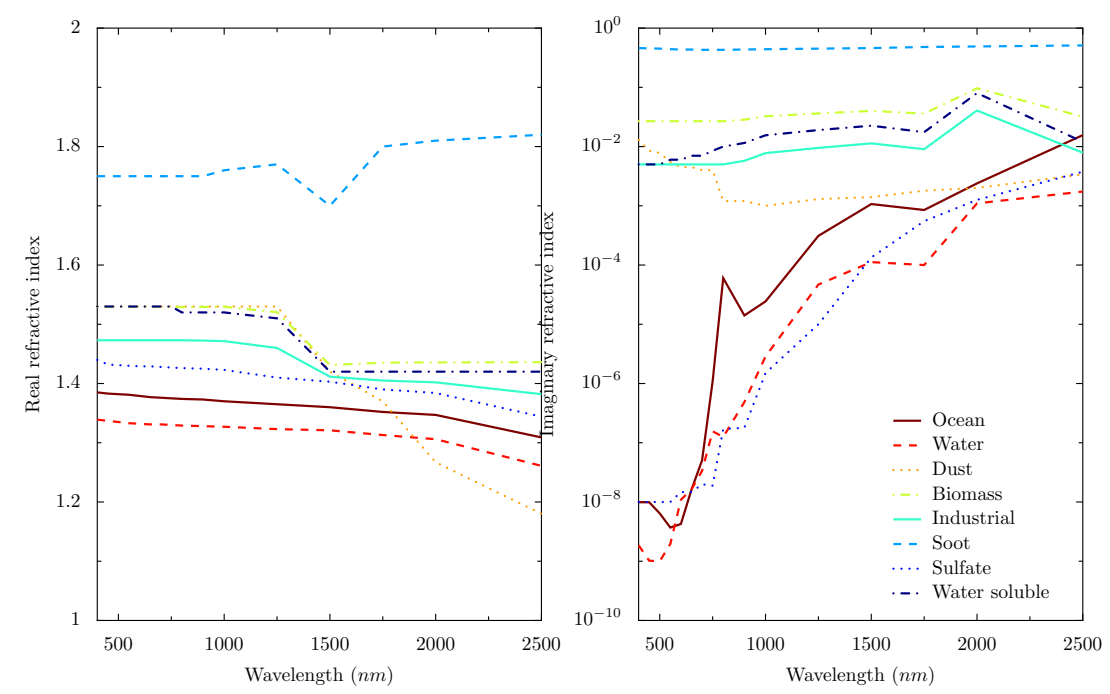

Figure 2. Imaginary (left panel) and real parts (right panel) of the refractive index as a function of wavelength. Data taken from D'Almeida et al. (1991).

\section{Methods}

\subsection{Forward model}

In the PODEX and SEAC ${ }^{4}$ RS campaigns, the airborne RSP measurements are performed at an altitude of about $20 \mathrm{~km}$. To model the radiance and state of polarization of light of a given wavelength measured at the RSP flight altitude, we consider a macroscopically isotropic, plane-parallel multilayered atmosphere bounded below by a reflecting surface. Each atmosphere layer is characterized by scattering and absorption optical thickness and the scattering phase matrix $\mathbf{P}$.

For RSP shortwave infrared bands, gaseous absorption need to be taken into account. The absorption in RSP 1590 and $2250 \mathrm{~nm}$ bands can be represented by a model that is derived using $k$ distributions for each $1 \mathrm{~nm}$ that are then integrated with a solar weighting over the RSP bandpasses. Here the total absorption optical depth depends on the total column optical depth of well-mixed gases and water vapor. It should be noted that water vapor absorption is relatively small when compared with that of the well-mixed gases $\mathrm{CO}_{2}$ at $1590 \mathrm{~nm}$ and methane at $2250 \mathrm{~nm}$. In terms of profile distributions, the integrated optical depths from the top of the atmosphere to a pressure level $p$ for the well-mixed gases and water vapor are proportional to $\left(p / p_{0}\right)^{2}$ and $\left(p / p_{0}\right)^{4}$, respectively, where $p_{0}$ is the surface pressure.

The optical properties of aerosols depend on the size, shape and type of aerosols. There is sufficient evidence that mixtures of spheroids allow rather accurate fitting of measured spectral and angular dependencies of observed intensity and polarization (Dubovik et al., 2006). Here we consider scattering properties of both spherical and spheroidal aerosol particles. The optical properties of spherical and spheroidal aerosol particles are calculated using the tabulated kernels of Dubovik et al. (2006). Here we describe particles as a mixture of spheres and spheroids with the aspect ratio distribution proposed by Dubovik et al. (2006). 

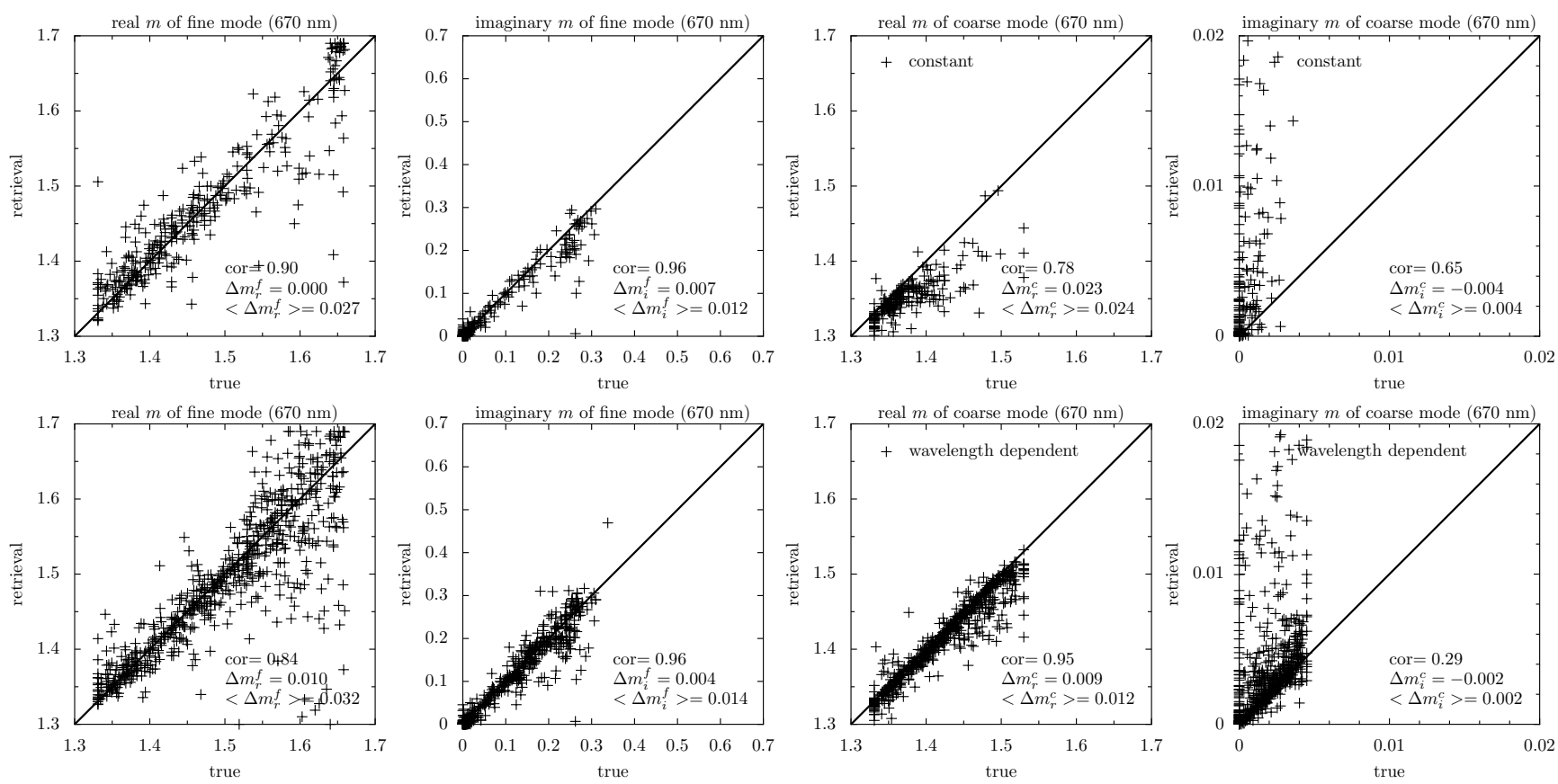

Figure 3. Comparison between accounting for (top panel, for $\chi^{2} \leq 2.0,35.3 \%$ cases converged) and not accounting for (bottom panel, $75.1 \%$ converged) spectral variation of refractive index in synthetic retrievals. The correlation coefficients (cor), mean error $(\Delta m)$ and mean absolute error $(<\Delta m\rangle)$ between retrieved and true values of refractive index are included.

For the characterization of surface reflection, the RahmanPinty-Verstraete model (RPV) (Rahman et al., 1993) is used with three independent parameters: $\rho_{0}, g$ and $k$ (see Appendix). The model successfully accounts for the observed variability of reflectance measurements in laboratory and field conditions, ranging from bare soil to full canopy cover, in both the visible and the near-infrared bands (Rahman et al., 1993). To account for surface polarized reflectance, we use a modified Fresnel model introduced by Litvinov et al. (2011) (see Appendix). It has been shown that the Bidirectional Reflectance and Polarization Distribution Function (BRDF and BPDF) used here can fit the RSP measurements with sufficient accuracy (Litvinov et al., 2011).

We solve the vector radiative transfer equation in the Earth atmosphere using the method as described by Hasekamp and Landgraf $(2002,2005 a)$. To solve the integration over azimuth angle, the radiative transfer equation is decomposed into a series of Fourier components. To reduce the number of terms needed in the Fourier series, the intensity vector originating from single scattering is calculated analytically. To efficiently treat highly peaked phase function we apply the "MS method" by Nakajima and Tanaka (1988). Then, an iterative Gauss-Seidel method is applied to solve the radiative transfer equations (Herman and Browning, 1965; Landgraf et al., 2001; Schepers et al., 2014).

\subsection{Retrieval method}

The aim of aerosol retrieval is to convert observed measurements into information about physical properties of aerosols, i.e.,

$\boldsymbol{y}=\mathbf{F}(\boldsymbol{x})+\boldsymbol{e}_{y}$.

Here $\boldsymbol{y}$ is the measurement vector that contains the multiplewavelength multiple-viewing angle measurements of radiance and degree of linear polarization (DOLP), $\mathbf{F}$ is the forward radiative transfer model and $\boldsymbol{e}_{y}$ is an error term. To define the state vector $\boldsymbol{x}$, we employ the bimodal lognormal function to describe the atmospheric aerosol size distributions including a fine and coarse mode indicated by superscripts $s$ and $c$. Here, each mode is characterized by the effective radius $r_{\text {eff }}$, the effective variance $v_{\text {eff }}$ and aerosolloading $N$ that is the number of aerosol particles per square meter. Spherical and spheroidal aerosol particles are distinguished by fraction ratio $p$, which indicates the percentage of spheroid particles. We consider a Gaussian height distribution $h(z)=A \exp \left(-4 \ln 2\left(z-z_{c}\right)^{2} / z_{w}\right)$ with center height $z_{c}$ and width $z_{w}$ for layer at height $z$ and include $z_{c}$ as one additional unknown parameter in our retrieval.

Additionally, the complex refractive index $m=m_{\mathrm{r}}+i m_{i}$, is needed to characterize aerosols of each mode. Figure 2 shows the spectral dependence of the most important aerosol types (D'Almeida et al., 1991). It can be seen in Fig. 2 that for the aerosol types that predominantly occur in the fine mode (biomass burning, industrial, soot, sulfate and water 

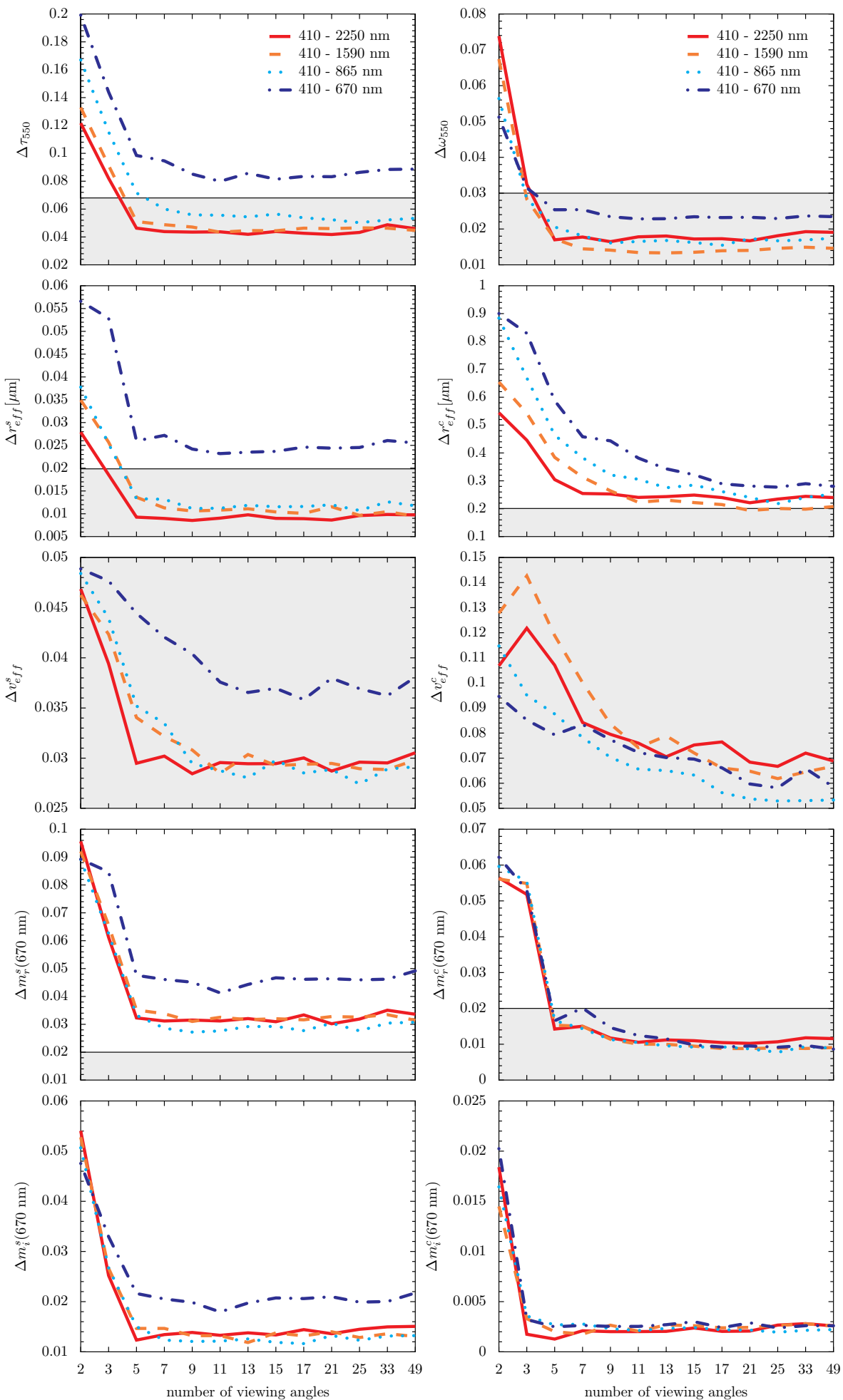

Figure 4. The results of mean absolute errors of aerosol optical depth $\left(\tau_{550}\right)$, single scattering albedo $\left(\omega_{550}\right)$, aerosol size parameters $\left(r_{\text {eff }}^{\mathrm{s}}\right.$, $v_{\mathrm{eff}}^{\mathrm{s}}, r_{\mathrm{eff}}^{\mathrm{c}}$ and $\left.v_{\mathrm{eff}}^{\mathrm{c}}\right)$ and refractive index $\left(m_{\mathrm{r}}^{\mathrm{s}}, m_{i}^{\mathrm{s}}, m_{\mathrm{r}}^{\mathrm{c}}\right.$ and $\left.m_{i}^{\mathrm{c}}\right)$ as a function of the number of viewing angles between retrieved and true values for four different RSP measurement configurations. Soil and vegetation surfaces are used in simulation. Aerosol types in Fig. 2 are all included in simulation and spectral variation of refractive index are included in both simulation and retrieval. The shaded areas indicate the target accuracy on aerosol parameters formulated by Mishchenko et al. (2004). For the target accuracy of AOD, we use $10 \%$ of the mean $\mathrm{AOD}$, which is 0.68 . 
soluble) the spectral dependence of the real part of the refractive index is relatively flat in the spectral range where they are most optically active $(<1200 \mathrm{~nm})$. Although the imaginary part of the refractive index for the fine mode types shows a spectral dependence for some types, the values for these types are generally small, so that such wavelength dependences will not significantly affect optical measurements in the visible spectral range. This may be different when "brown carbon"-type aerosols are included in the analysis (Feng et al., 2013). For the coarse mode the situation is different, since for this mode the real part of the refractive index generally shows a spectral dependence in the range $>\sim 1200 \mathrm{~nm}$ where coarse mode aerosols are optically active. Furthermore, for dust the imaginary part of the refractive index increases strongly in the blue/UV part of the spectrum. Here, the spectral refractive index of coarse mode aerosols are treated as a mixing of three classes refractive index spanning the wavelength range in Fig. 2 and thus can be described by a linear combination of them. If so, we have six parameters to be fit for both the real and imaginary part of refractive index of coarse mode aerosols. However, we find that these six parameters are strongly intercorrelated, so in fact less than six parameters are needed. The well-known principal component analysis method can be used to determine the most important spectral signatures in the refractive index (Smith, 2002). For the real part of complex refractive index, a $t \times n$ matrix $\mathbf{M}$ with $n \geq t$ is constructed as

$\mathbf{M}=\left[\boldsymbol{m}_{1}, \boldsymbol{m}_{2}, \cdots, \boldsymbol{m}_{t}\right]^{\mathrm{T}}$,

where $\mathrm{T}$ indicates the transposed vector and row vectors are the mean-subtracted real part of the refractive indices at $n$ different wavelengths for $t$ types of representative coarse mode aerosols. From the covariance matrix of $\mathbf{M}$, eigenvectors $\boldsymbol{m}_{i}^{d}$ can be derived in order of significance indicated by eigenvalues. We order the vector $\boldsymbol{m}_{i}^{d}$ according to their eigenvalues and map them into the original data space by multiplying with $\mathbf{M}$. Consequently, the original row vectors in matrix $\mathbf{M}$ or their linear combinations can be exactly reconstructed by wavelength-dependent vector $\boldsymbol{m}_{i}^{d}$ and wavelength-independent value $m^{\text {ind }}$ through

$\boldsymbol{m}=m^{\text {ind }}+\sum_{i=1}^{t^{\prime}} \alpha_{i} \cdot \boldsymbol{m}_{i}^{d}$,

where $t^{\prime}=t$ and $\alpha_{i}$ is the weight of vector $\boldsymbol{m}_{i}^{d}$. For coarse mode aerosols in Fig. 2, we found that for $t^{\prime}=1$ we can sufficiently describe the spectral features in the refractive index both for the real and the imaginary part.

In total, for coarse mode aerosols there are eight state vector elements of which two correspond to size ( $r_{\text {eff }}$ and $\left.v_{\text {eff }}\right)$, four correspond to refractive index $\left(m_{\mathrm{r}}^{\text {ind }}, \alpha_{\mathrm{r}}, m_{i}^{\text {ind }}\right.$ and $\left.\alpha_{i}\right)$ and two others correspond to aerosol-loading $N$ and spheroid fraction $p$, respectively. The fine mode aerosols are described in the same way with five state vector elements, i.e., without the parameters describing spectral dependence of the refractive index and the spheroid fraction. In the retrieval, we also retrieve the central height $z_{c}$ of the aerosol Gaussian height distribution. As a consequence, the state vector $\boldsymbol{x}$ contains 14 aerosol-related elements in total. In addition to these aerosol parameters we also include the surface reflection parameters $\rho_{0}, g$ and $k$ of RPV model and surface polarized reflectance parameters $\alpha$ and $\sigma$ of the modified Fresnel model in the state vector.

To retrieve the state vector $\boldsymbol{x}$ from RSP measurements, an inversion algorithm based on the Phillips-Tikhonov regularization is employed (Hasekamp and Landgraf, 2005b) which has been successfully applied to aerosol retrieval from PARASOL measurements (Hasekamp et al., 2011). The inversion algorithm chooses the solution $\hat{\boldsymbol{x}}$ that solves the minimization problem

$\hat{\boldsymbol{x}}=\min _{\boldsymbol{x}}\left(\left\|\mathbf{S}_{y}^{-\frac{1}{2}}(\mathbf{F}(\boldsymbol{x})-\boldsymbol{y})\right\|^{2}+\gamma\left\|\mathbf{W}\left(\boldsymbol{x}-\boldsymbol{x}_{\mathrm{a}}\right)\right\|^{2}\right)$,

where $\boldsymbol{x}_{\mathrm{a}}$ is a priori state vector, $\mathbf{W}$ is a weighting matrix and $\mathbf{S}_{y}$ is the measurement error covariance matrix that contains the measurement error estimate. The weighting matrix $\mathbf{W}$ is chosen diagonal such that $W_{i j}=1 / x_{\mathrm{a}, j}$, making the side constraint dimensionless. The regularization parameter $\gamma$ is found from a method similar to the $L$ curve (Hansen and O'Leary, 1993), in which we evaluate the least squares norm for different trial values of $\gamma$ using an approximate forward model and choose $\gamma$ for which the least squares norm is smallest.

To obtain an appropriate first guess state vector for the iterative retrieval procedure, we perform a retrieval using a lookup table based on 48 aerosol modes for the fine mode and 24 for the coarse mode. Table 2 shows the parameters of each mode.

For surface reflectance, the parameters of the RPV model used for generating the lookup table at each wavelength are shown in Table 3. For surface polarized reflectance, we use one mode of the modified Fresnel model in Table 3. The combination of fine and coarse mode and surface parameters that yields the best fit to the measurement is used as first guess and a priori in the full retrieval procedure.

\section{Aerosol retrieval}

\subsection{Synthetic retrieval}

First, we perform our investigations on a set of 1000 synthetic measurements in the principal plane at solar zenith angle $60.0^{\circ}$. Each synthetic measurement includes 49 equally sampled viewing angles $\left( \pm 60.0^{\circ}\right.$ from nadir) and seven of the nine RSP wavelength bands as described in Sect. 2. The relative azimuthal angles are 0.0 and $180.0^{\circ}$ for positive and negative viewing zenith angles, respectively. Here we use the convention that an azimuth angle of $180.0^{\circ}$ corresponds 

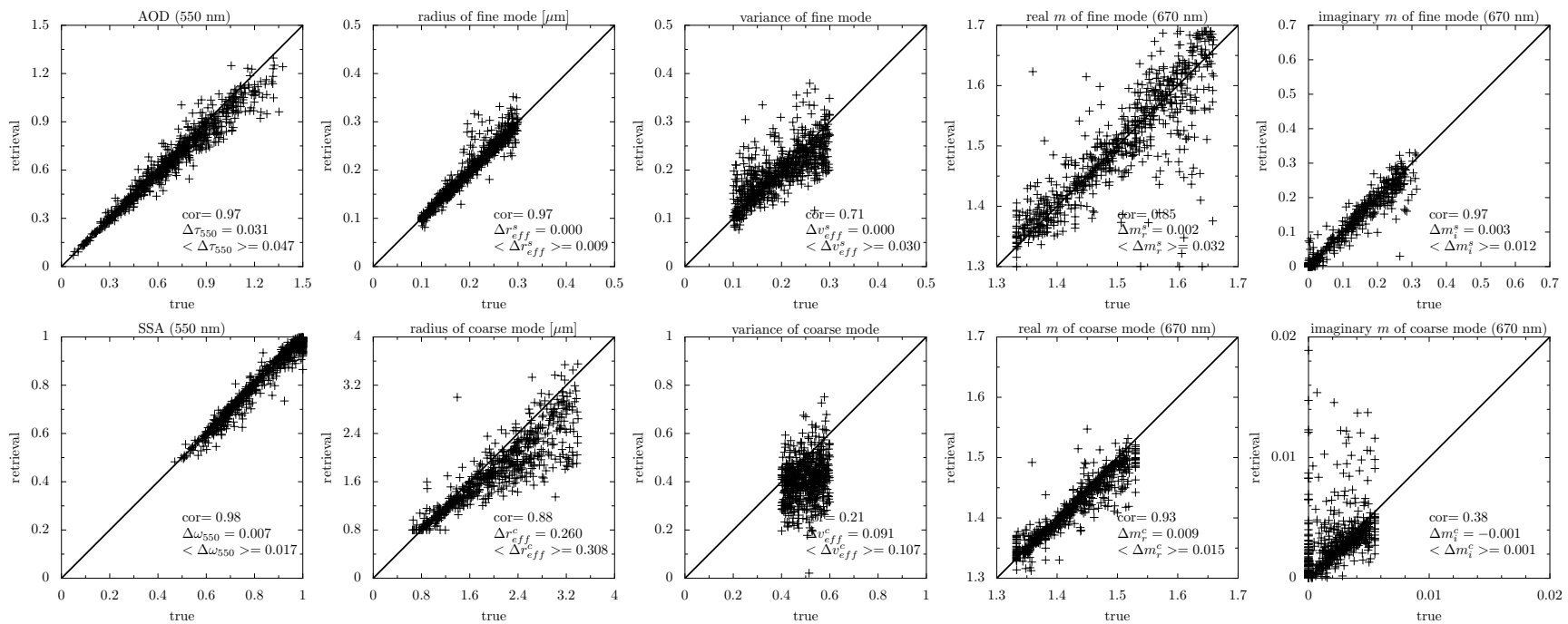

Figure 5. Comparison between retrieved and true values of aerosol optical depth $\left(\tau_{550}\right)$, single scattering albedo $\left(\omega_{550}\right)$ and aerosol parameters $\left(r_{\mathrm{eff}}^{\mathrm{s}}, v_{\mathrm{eff}}^{\mathrm{s}}, m_{\mathrm{r}}^{\mathrm{s}}, m_{\mathrm{i}}^{\mathrm{s}}, r_{\mathrm{eff}}^{\mathrm{c}}, v_{\mathrm{eff}}^{\mathrm{c}}, m_{\mathrm{r}}^{\mathrm{c}}\right.$ and $\left.m_{\mathrm{i}}^{\mathrm{c}}\right)$ when the viewing angle number $N=5$ using the full spectral range $410-2250 \mathrm{~nm}$. The correlation coefficients (cor), mean error $(\Delta)$ and mean absolute error $(<\Delta>)$ are included.

Table 2. Parameters of fine and coarse modes used in lookup table retrieval.

\begin{tabular}{lcccccc}
\hline Modes & $r_{\text {eff }}[\mu \mathrm{m}]$ & $v_{\text {eff }}$ & $m_{\mathrm{r}}$ & $m_{i}$ & $p$ & $\tau$ \\
\hline Fine mode & $0.05,0.10,0.20,0.30$ & 0.19 & $1.37,1.45,1.52,1.60$ & $0.001,0.020,0.100$ & 1.0 & $0.01,0.15,0.25,0.5,0.8,1.0,1.5,3.0,5.0$ \\
Coarse mode & $1.00,2.50$ & 0.43 & $1.38,1.44,1.50$ & $0.001,0.050$ & $0.1,1.0$ & \\
\hline
\end{tabular}

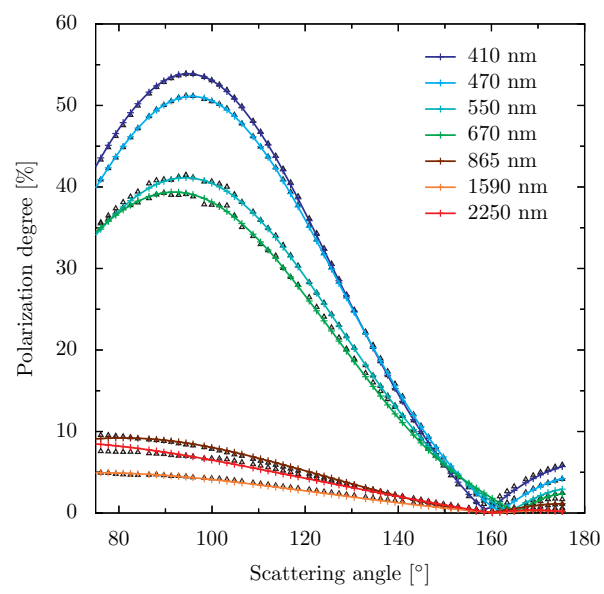

Figure 6. One example of RSP measurements and the corresponding fit results for DOLP in retrieval. Triangle symbols denote the RSP measurements and solid lines with plus are fits using the forward model in Sect. 3. The date, AERONET site (longitude, latitude), number of viewing angles, AOD, mean angular deviation from the principal plane and solar zenith angle are 9 September 2013, Baskin $\left(91.73^{\circ} \mathrm{W}, 32.28^{\circ} \mathrm{N}\right), 60,0.27(440 \mathrm{~nm}), 1.99^{\circ}$ and $66.5^{\circ}$, respectively. to backward scattering. To get measurement configurations with different angular resolution, viewing angles are evenly sampled from -60.0 to $60.0^{\circ}$ according to the resolution except in the measurement configurations with two viewing angles for which we use nadir and $-60.0^{\circ}$. Aerosol size distribution parameters used here are randomly distributed in their acceptable range for both fine mode $\left(0.1 \leq r_{\text {eff }}^{\mathrm{s}} \leq 0.30 \mu \mathrm{m}\right.$, $\left.0.1 \leq v_{\text {eff }}^{\mathrm{s}} \leq 0.30\right)$ and coarse mode $\left(0.60 \leq r_{\text {eff }}^{\mathrm{c}} \leq 3.50 \mu \mathrm{m}\right.$, $0.4 \leq v_{\mathrm{eff}}^{\mathrm{c}} \leq 0.6$ ). To account for the spectral dependence, the refractive indices for each mode are linear combinations of the refractive index spectra as shown in Fig. 2. The AOD $\tau$ for each mode varies randomly in the range $0.0<\tau_{550} \leq$ 0.70 . Aerosol layer center height are fixed to be $2000 \mathrm{~m}$ with a width of $2000 \mathrm{~m}$. We consider soil and vegetation land surfaces of RPV model and the modified Fresnel model with parameters presented in Table 4 (Litvinov et al., 2011). Parameters $\rho_{0}$ at $410,470,550$ and $865 \mathrm{~nm}$ are obtained by linear interpolation using soil and vegetation surface reflectance provided by ASTER spectral library (Baldridge et al., 2009).

The forward model as described in Sect. 3 was used to simulate the intensity vector at the altitude of RSP $(20 \mathrm{~km})$ for all 1000 combinations of input parameters. In our simulations, we add an random noise of $2.0 \%$ on intensity $I$ and $0.2 \%$ on DOLP $P$ to account for measurement uncertainties. 
Table 3. Parameters of RPV model and the modified Fresnel model used in lookup table retrieval.

\begin{tabular}{lccc}
\hline$\lambda[\mathrm{nm}]$ & $\rho_{0}$ & $g$ and $k$ & $\alpha, \sigma$ and $k_{\gamma}$ \\
\hline 410 & $0.02,0.04,0.06,0.10,0.15$ & & \\
470 & $0.02,0.05,0.08,0.15,0.25$ & $g=-0.1, k=0.2$ or $g=0.5, k=1.0$ & $\alpha=1.0, \sigma=2.0$ and $k_{\gamma}=0.69$ \\
Other bands & $0.10,0.15,0.20,0.30,0.45$ & & \\
\hline
\end{tabular}
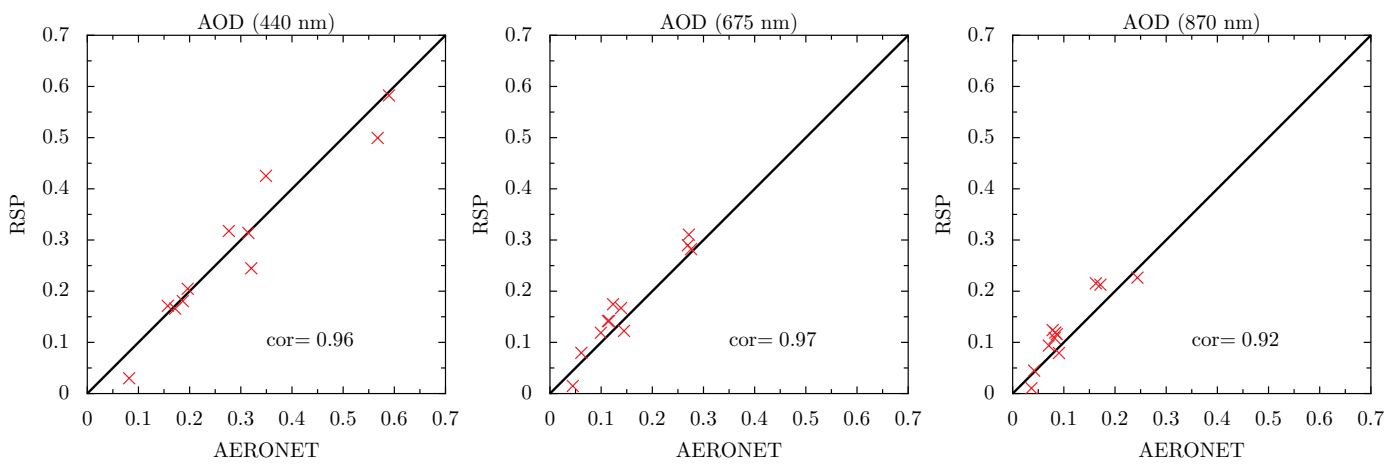

Figure 7. Comparison between the retrieved AOD of RSP measurements and AERONET at 440, 675 and $870 \mathrm{~nm}$. The correlation coefficients (cor) at each wavelength are included.
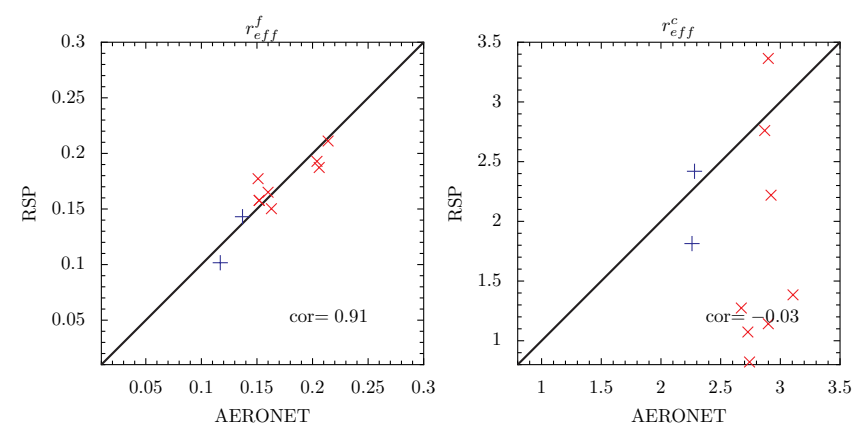

Figure 8. Comparison between the retrieved fine and coarse modes effective radius $\left(r_{\text {eff }}^{\mathrm{f}}\right.$ and $\left.r_{\text {eff }}^{\mathrm{c}}\right)$ of RSP measurements and AERONET. The correlation coefficients (cor) for each mode are included. Plus indicates cases where $\tau_{675}<0.1$.

\subsubsection{Spectral dependent refractive index}

We first test the effect of spectral variation of the refractive index in the retrieval. A measurement configuration is used with a wavelength range of $410-2250 \mathrm{~nm}$ and 13 viewing angles. We consider retrievals to be successfully converged if the $\chi^{2}$ of the fit $\leq 2.0$ with $\chi^{2}$ defined as $\frac{1}{N} \sum_{i=1}^{N}\left(\frac{y_{i}-F_{i}}{\sigma_{i}}\right)^{2}$, in which $N$ is the number of measurements, $y_{i}$ is the measurement, $F_{i}$ is the simulated result and $\sigma_{i}$ is the error of the measurement. We found that this $\chi^{2}$ threshold yields a reasonable balance between accuracy and number of converged cases. Figure 3 compares retrieved refractive indices (at $670 \mathrm{~nm}$ ) of fine and coarse modes with true values for retrievals using either a constant or a wavelength-dependent refractive index. The general trend is that for fine mode aerosols, as expected, both approaches yield similar results; for coarse mode aerosols, considering the spectral variation improves the agreement between retrieved and true values for both the real and the imaginary part of the refractive index. Most importantly, the number of retrievals that converge with a sufficiently good fit to the measurement $\left(\chi^{2} \leq 2.0\right)$ more than doubles from 35.3 to $75.1 \%$.

\subsubsection{Comparison between measurement configurations}

To investigate the importance of spectral range and angular resolution, we investigate retrieval errors on $\tau_{550}$ (AOD), $\omega_{550}$ (single scattering albedo, SSA), $r_{\mathrm{eff}}^{\mathrm{s}}, v_{\mathrm{eff}}^{\mathrm{s}}, r_{\mathrm{eff}}^{\mathrm{c}}, v_{\mathrm{eff}}^{\mathrm{c}}, m_{\mathrm{r}}^{\mathrm{s}}$, $m_{i}^{\mathrm{s}}, m_{\mathrm{r}}^{\mathrm{c}}$ and $m_{i}^{\mathrm{c}}$ using different measurement configurations. Here we focus on four different wavelength ranges, namely $410-670,410-865,410-1590$ and $410-2250 \mathrm{~nm}$. The number of viewing angles changes between 2 and 49. Although the number of cases that converge to $\chi^{2} \leq 2.0$ may change per measurement configuration, we slightly adjust the $\chi^{2}$ threshold for each measurement configuration so that the convergence percentage is $75 \%$ for all of them.

Figure 4 shows the results of mean absolute errors of each parameter as a function of the number of viewing angles $(N)$ for the four wavelength ranges. It is important to note that the results remain virtually unchanged when we use a different realization of 1000 combinations of aerosol parameters or when we increase the number of realizations. The shaded areas indicate the target accuracy on aerosol parameters formulated by Mishchenko et al. (2004). First of all, for all four wavelength ranges the retrieval errors are significantly decreased when the number of viewing angles $N$ is 
Table 4. Parameters of RPV model and the modified Fresnel model used in synthetic retrieval.

\begin{tabular}{|c|c|c|c|c|c|c|c|c|}
\hline \multirow[b]{2}{*}{ Surface types } & \multicolumn{4}{|c|}{ RPV model } & & \multicolumn{3}{|c|}{ Modified Fresnel model } \\
\hline & $\rho_{0}$ & $g$ & $k$ & & $\alpha$ & $\sigma$ & $k_{\gamma}$ & \\
\hline Soil & $\begin{array}{l}0.001(410 \mathrm{~nm}), \\
0.014(550 \mathrm{~nm}), \\
0.106(865 \mathrm{~nm}), \\
0.116(2250 \mathrm{~nm})\end{array}$ & $\begin{array}{l}0.007(470 \mathrm{~nm}), \\
0.071(670 \mathrm{~nm}), \\
0.159(1590 \mathrm{~nm}),\end{array}$ & -0.097 & 0.746 & & 4.260 & 0.589 & 0.700 \\
\hline Vegetation & $\begin{array}{l}0.013(410 \mathrm{~nm}), \\
0.024(550 \mathrm{~nm}), \\
0.120(865 \mathrm{~nm}), \\
0.060(2250 \mathrm{~nm})\end{array}$ & $\begin{array}{l}0.017(470 \mathrm{~nm}), \\
0.034(670 \mathrm{~nm}), \\
0.128(1590 \mathrm{~nm}),\end{array}$ & -0.071 & 0.725 & & 2.707 & 0.648 & 0.700 \\
\hline
\end{tabular}
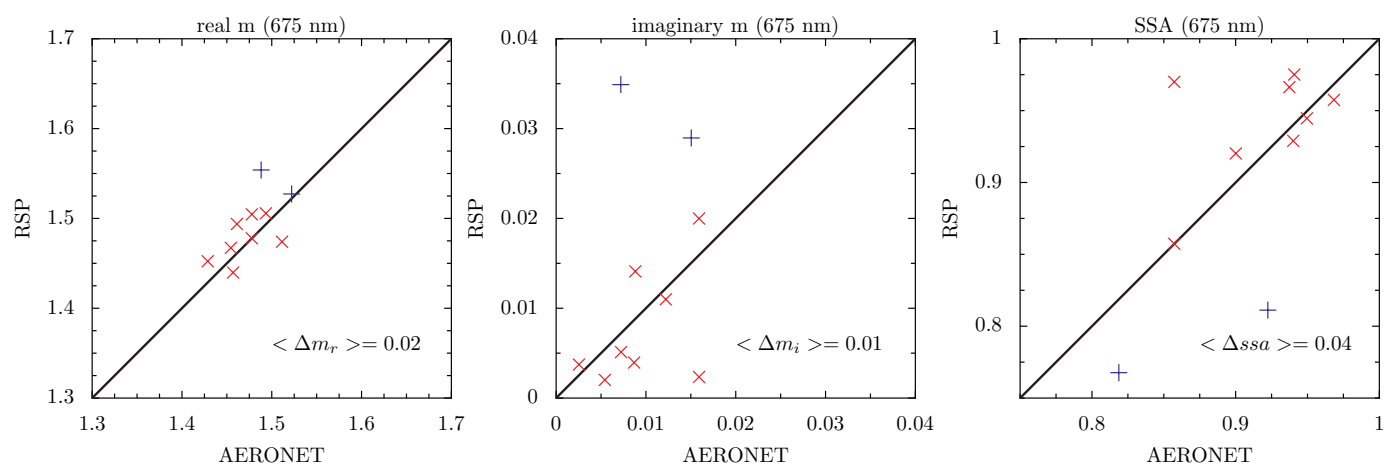

Figure 9. Comparison between retrieved single scattering albedo (SSA) and composed refractive index of RSP measurements and those provided by AERONET at $675 \mathrm{~nm} .\langle\Delta \mathrm{ssa}\rangle,\left\langle\Delta m_{\mathrm{r}}\right\rangle$ and $\left\langle\Delta m_{i}\right\rangle$ are the mean absolute difference of SSA and real and imaginary part of the refractive index between RSP and AERONET. Plus indicates cases where $\tau_{675}<0.1$.

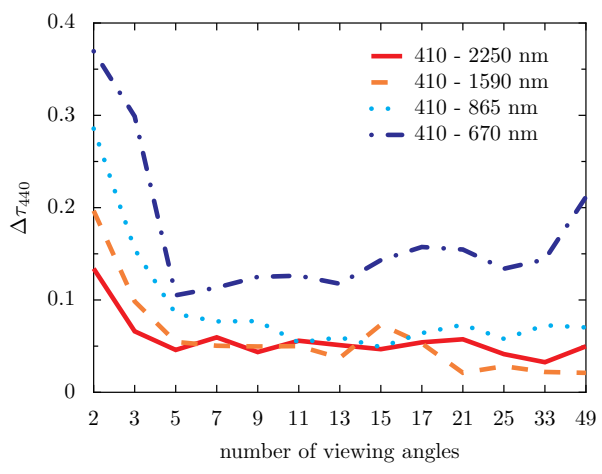

Figure 10. Retrieved AOD mean absolute error dependence on number of viewing angles for different spectral ranges of RSP data.

increased from 2 to 3 . The decrease after $N \geq 3$ is moderate in compared with the change from 2 to 3 . The mean errors do not improve significantly when the number $N$ is greater than $\sim 5$. Moreover, Fig. 4 also shows the advantage of the measurement configurations with spectral ranges 410-1590 and $410-2250 \mathrm{~nm}$, as they yield smaller retrieval errors than measurement configurations with a smaller spectral range. When compared with the $410-670$ and $410-865 \mathrm{~nm}$ cases, the mean error on the AOD at $550 \mathrm{~nm} \tau_{550}$ is reduced from $\sim 0.1$ and $\sim 0.06$ to $\sim 0.04$ as shown in the upper left figure. The mean error on the SSA at $550 \mathrm{~nm} \omega_{550}$ is less than 0.02 , while for the $410-670 \mathrm{~nm}$ case the errors are around 0.03 . The measurements of 410-1590 and 410-2250 nm yield smaller errors especially for the coarse mode. For example, the mean error on the effective radius $r_{\text {eff }}^{\mathrm{c}}$ is reduced from $\sim 0.3$ to $\sim 0.2 \mu \mathrm{m}$ and the mean error on the real and imaginary part of the refractive index is also reduced. This is attributable to the fact that shortwave infrared bands are sensitive to coarse mode aerosols. For fine mode aerosols, the measurements of $410-865 \mathrm{~nm}$ perform almost equally well as retrievals including shortwave infrared bands. Finally, the $410-670 \mathrm{~nm}$ case usually produces the largest mean error for all parameters. In contrast, the $410-2250 \mathrm{~nm}$ case can meet the target accuracy requirements indicated by shaded area for nearly all parameters. When we compare retrievals in the range $410-1590 \mathrm{~nm}$ with retrievals in the range $410-2250 \mathrm{~nm}$, we see that the accuracy of the aerosol optical depth retrievals is only minimally reduced with the latter setup, while for the other parameters the performance is similar.

Figure 5 shows scatterplots of retrieved vs. true values for $\tau_{550}, \omega_{550}$ and parameters of both aerosol modes when the viewing angle number $N=5$ using the full spectral range 
410-2250 nm. The correlation coefficients between true and retrieved values for each parameter are $0.97\left(\tau_{550}\right), 0.98$ $\left(\omega_{550}\right), 0.97$ (fine mode effective radius $r_{\text {eff }}^{\mathrm{s}}$ ), 0.71 (fine mode effective variance $v_{\text {eff }}^{\mathrm{s}}$ ), 0.85 (fine mode real part of the refractive index $m_{\mathrm{r}}^{\mathrm{s}}$ ), 0.97 (fine mode imaginary part of the refractive index $m_{i}^{\mathrm{s}}$ ), 0.88 (coarse mode effective radius $r_{\text {eff }}^{\mathrm{c}}$ ), 0.21 (coarse mode effective variance $v_{\text {eff }}^{\mathrm{c}}$ ), 0.93 (coarse mode real part of the refractive index $m_{\mathrm{r}}^{\mathrm{c}}$ ) and 0.38 (coarse mode imaginary part of the refractive index $m_{i}^{\mathrm{c}}$ ). The retrievals of the imaginary part of the coarse model aerosol can be poor when fine mode aerosols dominate. However, as shown in Fig. 5, the retrieved single scattering albedo values agree well with true values, which indicates good retrievals on total absorption.

\subsection{Aerosol retrieval using the RSP data}

We applied our algorithm described above to RSP data over land close (distance $\leq 20 \mathrm{~km}$ ) to AERONET stations so that we can compare our results with independent measurements. The data were pre-processed as described in Sect. 2. Retrievals are made only on cloud-free measurements because clouds are not included in the forward model. Time differences between the RSP measurements and AERONET products are restricted to less than $1 \mathrm{~h}$. We use the level 2 direct sun products for AOD comparison and level 1.5 inversion products for refractive index and single scattering albedo comparison. In the AERONET products, aerosol optical depth values are provided at wavelengths from 340 to $1640 \mathrm{~nm}$ with an estimated uncertainty of $0.01-0.02$, which are very well suited for validation of the AOD retrieved by RSP.

For the aerosol properties from the AERONET inversion product it should be noted that the corresponding accuracies are less well quantified and expected to be similar or larger than those of the RSP retrievals. So, for SSA, refractive index and effective radius, the results presented below should be considered as a comparison rather than a validation. Apart from this, AERONET retrieves one refractive index whereas we retrieve refractive indices for both the fine and coarse mode. To get a general impression on the agreement between AERONET and RSP retrieval, we compose a refractive index $m_{\text {comp }}$ that is a weighted average by AOD $\tau$ of each mode:

$m_{\text {comp }}=\frac{m^{\mathrm{s}} \tau_{\mathrm{s}}+m^{\mathrm{c}} \tau_{\mathrm{c}}}{\tau_{\mathrm{s}}+\tau_{\mathrm{c}}}$.

The weighted refractive index $m_{\text {comp }}$ should reflect to some extent the retrieval capability on this quantity.

In our RSP retrieval, we use five viewing angles which are evenly sampled within the available range of each measurement and consider cases that passed a goodness of fit criterion under $\chi^{2} \leq 10.0$. The $\chi^{2}$ threshold here is larger than the one we used in synthetic retrieval, because some measurements are still hampered by angular oscillations caused by inhomogeneity of the underlying surface or atmosphere.
Besides this, we include only cases where the sampled scattering range includes the $85.0^{\circ}-155.0^{\circ}$ interval because retrieval errors are considerably larger for smaller angular ranges (Hasekamp and Landgraf, 2007). Figure 6 shows one example of RSP measurements and the corresponding fit results for DOLP. Generally, the fits are good for each spectral band. In some cases, the measurements can be found to fluctuate between some viewing angles, thus making the fits worse (see the fit for $670 \mathrm{~nm}$ in Fig. 6).

Figure 7 shows a comparison between the retrieved AOD from RSP measurements and the AOD provided by AERONET at 440,675 and $870 \mathrm{~nm}$. Here we show retrieval results using the wavelength range $410-1590 \mathrm{~nm}$. From Fig. 7 it follows that retrieved AODs at the three wavelengths are in good agreement with the AERONET results. The correlation coefficients are 0.96 for $440 \mathrm{~nm}, 0.97$ for $675 \mathrm{~nm}$ and 0.92 for $870 \mathrm{~nm}$.

In Fig. 8 the retrieved effective radii of fine mode aerosol agree well with those of AERONET. The bias in coarse mode is mainly attributable to the fact that the retrieved AODs of the coarse mode are relatively small (mostly less than 0.1), which makes the retrieved values less reliable.

Figure 9 shows the values of the SSA, refractive index $m$ and the corresponding AERONET values at $675 \mathrm{~nm}$. For the SSA, the retrieval results agree reasonably well with AERONET values. The mean absolute difference in SSA between AERONET and RSP is 0.04. Under the assumption that the AERONET refractive index can be approximated by the weighted average of the two modes, the retrieved values are relatively close to those provided by AERONET. As expected, the deviation between RSP and AERONET is larger for cases with small AOD $\left(\tau_{675}<0.1\right)$ as indicated by the color code in Figure 9.

The retrieved center heights $z_{c}$ varied in the range of 0.97 and $2.66 \mathrm{~km}$ and for each case a similar height range is retrieved by the Cloud Physics Lidar (CPL) (not shown). A more extensive comparison between retrieved heights from RSP and those from CPL will be subject to future investigation.

Figure 10 shows the difference in AOD between RSP and AERONET as a function of the number of viewing angles used in the retrieval. Due to the limited number of cases for which AERONET SSA and refractive index measurements are available, we restrict the analysis to AOD only. We should notice that with the increase of $N$ the retrieval performance sometimes becomes even a bit worse, which is due to the fact that the measurements at some viewing directions can be distorted by the inhomogeneity of the land surface. In general, the results of Fig. 10 confirm our findings from the study with synthetic measurements: (1) the strongest improvement in accuracy is seen when $N$ increases from 2 to 3; (2) the retrieval performances are not significantly further improved if more than five viewing angles are used in the retrieval; (3) retrievals perform significantly better when shortwave infrared bands are included. 


\section{Conclusions}

In this paper we performed aerosol retrievals over land from multiangle photopolarimetric measurements produced synthetically and obtained by the airborne RSP instrument. We adjusted the SRON aerosol retrieval algorithm to cope with spectrally dependent refractive indices so that it can be applied to all RSP spectral channels.

We investigated the capability of different retrieval setups with varying spectral ranges and angular resolutions by considering 1000 synthetic measurements for different aerosol scenarios. We find that the errors on the retrieved parameters were significantly decreased when including multiple viewing geometries. When more than five viewing angles are included in the retrieval, virtually no significant improvement was found anymore. It should be noted that for future instrumentation a higher number of viewing angles is still desirable in order to separate scattering by aerosols and cloud droplets by means of the rainbow (Waquet et al., 2009b; Hasekamp, 2010) and for the retrieval of cloud microphysical quantities (Alexandrov et al., 2012). Moreover, we demonstrated the advantage of including $1590 \mathrm{~nm}$ shortwave infrared bands in the retrieval over retrievals restricted to the visible spectral range. This improvement is in particular significant for coarse mode aerosol parameters such as effective radius and refractive index. Here, the spectral range 410-1590 and 410$2250 \mathrm{~nm}$ showed similar performance.
For retrievals from real RSP measurements, the retrieved AOD agrees well with AERONET products. The correlation coefficients are $0.96(440 \mathrm{~nm}), 0.97(675 \mathrm{~nm})$ and $0.92(870 \mathrm{~nm})$. The retrieved SSA also agrees well with AERONET although it should be noted that the number of comparison points is limited. The retrieved refractive index averaged over the two modes is consistent with the AERONET refractive index. The retrieval results of RSP measurements with different spectral ranges and angular resolutions confirm the findings of the synthetic study, i. e., that five viewing angles is sufficient for aerosol retrievals and that the inclusion of measurements at $1590 \mathrm{~nm}$ is important. 
Appendix: Models for surface reflection of radiance and polarized radiance

The Rahman-Pinty-Verstraete model is used with the following form:

$R_{I}\left(\lambda, \vartheta_{\mathrm{v}}, \vartheta_{0}, \phi\right)=\frac{\left(\cos \vartheta_{0} \cos \vartheta_{\mathrm{v}}\right)^{k-1}}{\left(\cos \vartheta_{0}+\cos \vartheta_{\mathrm{v}}\right)^{1-k}} \rho_{0}(\lambda) F(\gamma)$

$(1+R(G))$

$F(\gamma)=\frac{1-g^{2}}{\left(1+g^{2}-2 g \cos \gamma\right)^{1.5}}$,

$1+R(G)=1+\frac{1-\rho_{0}}{1+G}$,

$G=\sqrt{\tan ^{2} \vartheta_{0}+\tan ^{2} \vartheta_{\mathrm{v}}-2 \tan \vartheta_{0} \tan \left|\vartheta_{\mathrm{v}}\right| \cos \phi}$.

Here $\rho_{0}, g$ and $k$ are three independent parameters; $\lambda$ is wavelength; $\gamma$ is scattering angle; $\phi$ is the difference of azimuth angles, $\phi=\phi_{\mathrm{v}}-\phi_{0} ; \vartheta_{\mathrm{v}}$ and $\vartheta_{0}$ are viewing and solar zenith angles, respectively; $F(\gamma)$ is Henyey-Greenstein phase function; $1+R(G)$ is an approximation of the hot spot effect.
The modified Fresnel model is written as

$$
\begin{aligned}
& R_{\mathrm{p}}\left(\vartheta_{\mathrm{v}}, \vartheta_{0}, \phi\right)=\frac{\alpha \pi F_{\mathrm{p}}(m, \gamma)}{4 \boldsymbol{u}_{n}\left(\cos \vartheta_{\mathrm{v}}+\cos \vartheta_{0}\right)} f\left(\boldsymbol{n}_{\mathrm{v}}, \boldsymbol{n}_{0}\right) f_{\mathrm{sh}}(\gamma), \\
& f\left(\boldsymbol{n}_{\mathrm{v}}, \boldsymbol{n}_{0}\right)=\frac{1}{\pi \mu_{n}^{3} 2 \sigma^{2}} \exp \left(-\frac{1-\mu_{n}^{2}}{\mu_{n}^{2} 2 \sigma^{2}}\right) \\
& f_{\mathrm{sh}}(\gamma)=\left(\frac{1+\cos k_{\gamma}(\pi-\gamma)}{2}\right)^{3} .
\end{aligned}
$$

Here $f\left(\boldsymbol{n}_{\mathrm{v}}, \boldsymbol{n}_{0}\right)$ is a Gaussian function which describes the distribution of facets over orientation, where $\sigma$ is the standard deviation of surface facet slopes. $f_{\mathrm{sh}}(\gamma)$ is a shadowing function with free parameter $k_{\gamma} . F_{p}(m, \gamma)$ is the element $F_{21}$ of the Fresnel scattering matrix with refractive index $m . \alpha$ is a scaling parameter. $\boldsymbol{n}_{0}$ and $\boldsymbol{n}_{\mathrm{v}}$ are vectors with form $\left(\sin \vartheta_{0} \cos \phi_{0}, \sin \vartheta_{0} \sin \phi_{0}, \cos \phi_{0}\right)$ and $\left(\sin \left|\vartheta_{\mathrm{v}}\right| \cos \phi_{\mathrm{v}}, \sin \left|\vartheta_{\mathrm{v}}\right| \sin \phi_{\mathrm{v}}, \cos \phi_{\mathrm{v}}\right) . \boldsymbol{u}_{\mathrm{n}}$ is defined by $\frac{n_{\mathrm{v}}^{z}+n_{0}^{z}}{\left|\boldsymbol{n}_{\mathrm{v}}+\boldsymbol{n}_{0}\right|}$, where $n_{\mathrm{v}}^{z}$ and $n_{0}^{z}$ are $z$ components of the vector $\boldsymbol{n}_{\mathrm{v}}$ and $\boldsymbol{n}_{0}$. 
Acknowledgements. We wish to thank two anonymous reviewers, Matteo Ottaviani, Thibaut Lurton, and Michael Garay for their interest and valuable comments which have led to several improvements. The RSP data from the SEAC ${ }^{4} \mathrm{RS}$ and PODEX field experiments that are used in this study were funded by the NASA Radiation Sciences Program managed by Hal Maring and by the NASA Earth Science Division as part of the pre-formulation study for the Aerosol Cloud and ocean Ecosystem (ACE) mission. L. Wu was supported by a grant from the China Scholarship Council (201306690014).

Edited by: A. Kokhanovsky

\section{References}

Alexandrov, M. D., Cairns, B., Emde, C., Ackerman, A. S., and van Diedenhoven, B.: Accuracy assessments of cloud droplet size retrievals from polarized reflectance measurements by the research scanning polarimeter, Remote Sens. Environ., 125, 92111, 2012.

Baldridge, A., Hook, S., Grove, C., and Rivera, G.: The aster spectral library version 2.0, Remote Sens. Environ., 113, 711-715, 2009.

Cairns, B., Russell, E. E., and Travis, L. D.: Research scanning polarimeter: calibration and ground-based measurements, in: SPIE's International Symposium on Optical Science, Engineering, and Instrumentation, International Society for Optics and Photonics, 186-196, 1999.

D'Almeida, G. A., Koepke, P., and Shettle, E. P.: Atmospheric Aerosols: Global Climatology and Radiative Characteristics, A. Deepak Pub., Hampton, 1991.

Dubovik, O., Sinyuk, A., Lapyonok, T., Holben, B. N., Mishchenko, M., Yang, P., Eck, T. F., Volten, H., Munoz, O., Veihelmann, B., Zande, v. d. W. J., Leon, J.-F., Sorokin, M., and Slutsker, I.: Application of spheroid models to account for aerosol particle nonsphericity in remote sensing of desert dust, J. Geophys. Res.Atmos., 111, D11208, doi:10.1029/2005JD006619, 2006.

Dubovik, O., Herman, M., Holdak, A., Lapyonok, T., Tanré, D., Deuzé, J., Ducos, F., Sinyuk, A., and Lopatin, A.: Statistically optimized inversion algorithm for enhanced retrieval of aerosol properties from spectral multi-angle polarimetric satellite observations, Meas. Tech.+, 4, 975-1018, 2011.

Feng, Y., Ramanathan, V., and Kotamarthi, V. R.: Brown carbon: a significant atmospheric absorber of solar radiation?, Atmos. Chem. Phys., 13, 8607-8621, doi:10.5194/acp-13-8607-2013, 2013.

Hansen, P. C. and O'Leary, D. P.: The use of the l-curve in the regularization of discrete ill-posed problems, SIAM J. Sci. Comput., 14, 1487-1503, 1993.

Hasekamp, O. P.: Capability of multi-viewing-angle photopolarimetric measurements for the simultaneous retrieval of aerosol and cloud properties, Atmos. Meas. Tech., 3, 839-851, doi:10.5194/amt-3-839-2010, 2010.

Hasekamp, O. P. and Landgraf, J.: A linearized vector radiative transfer model for atmospheric trace gas retrieval, J. Quant. Spectrosc. Ra., 75, 221-238, 2002.
Hasekamp, O. P. and Landgraf, J.: Linearization of vector radiative transfer with respect to aerosol properties and its use in satellite remote sensing, J. Geophys. Res.-Atmos., 110, D04203, doi:10.1029/2004JD005260, 2005a.

Hasekamp, O. P. and Landgraf, J.: Retrieval of aerosol properties over the ocean from multispectral single-viewing-angle measurements of intensity and polarization: retrieval approach, information content, and sensitivity study, J. Geophys. Res.-Atmos., 110, D20207, doi:10.1029/2005JD006212, 2005b.

Hasekamp, O. P. and Landgraf, J.: Retrieval of aerosol properties over land surfaces: capabilities of multiple-viewing-angle intensity and polarization measurements, Appl. Optics, 46, 33323344, 2007.

Hasekamp, O. P., Litvinov, P., and Butz, A.: Aerosol properties over the ocean from parasol multiangle photopolarimetric measurements, J. Geophys. Res.-Atmos., 116, D14204, doi:10.1029/2010JD015469, 2011.

Herman, B. M. and Browning, S. R.: A numerical solution to the equation of radiative transfer, J. Atmos. Sci., 22, 559-566, 1965.

IPCC: Climate change 2013: the physical science basis, Intergovernmental Panel on Climate Change, Working Group I Contribution to the IPCC Fifth Assessment Report (AR5), Cambridge Univ. Press, New York, 2013.

Kistler, R., Kalnay, E., Collins, W., Saha, S., White, G., Woollen, J., Chelliah, M., Ebisuzaki, W., Kanamitsu, M., Kousky, V., Dool, v. d. H., Jenne, R., and Fiorino, M.: The ncep-ncar 50-year reanalysis: monthly means cd-rom and documentation, B. Am. Meteorol. Soc., 82, 247-267, 2001.

Knobelspiesse, K., Cairns, B., Mishchenko, M., Chowdhary, J., Tsigaridis, K., van Diedenhoven, B., Martin, W., Ottaviani, M., and Alexandrov, M.: Analysis of fine-mode aerosol retrieval capabilities by different passive remote sensing instrument designs, Opt. Express, 20, 21457-21484, 2012.

Landgraf, J., Hasekamp, O. P., Box, M. A., and Trautmann, T.: A linearized radiative transfer model for ozone profile retrieval using the analytical forward-adjoint perturbation theory approach, J. Geophys. Res.-Atmos., 106, 27291-27305, 2001.

Litvinov, P., Hasekamp, O. P., and Cairns, B.: Models for surface reflection of radiance and polarized radiance: comparison with airborne multi-angle photopolarimetric measurements and implications for modeling top-of-atmosphere measurements, Remote Sens. Environ., 115, 781-792, 2011.

Mishchenko, M. I. and Travis, L. D.: Satellite retrieval of aerosol properties over the ocean using polarization as well as intensity of reflected sunlight, J. Geophys. Res.-Atmos., 102, 1698917013, 1997.

Mishchenko, M. I., Cairns, B., Hansen, J. E., Travis, L. D. Burg, R., Kaufman, Y. J., Vanderlei Martins, J., and Shettle, E. P.: Monitoring of aerosol forcing of climate from space: analysis of measurement requirements, J. Quant. Spectrosc. Ra., 88, 149-161, 2004.

Nakajima, T. and Tanaka, M.: Algorithms for radiative intensity calculations in moderately thick atmospheres using a truncation approximation, J. Quant. Spectrosc. Ra., 40, 51-69, 1988.

Ottaviani, M., Knobelspiesse, K., Cairns, B., and Mishchenko, M.: Information content of aerosol retrievals in the sunglint region, Geophys. Res. Lett., 40, 631-634, 2013.

Rahman, H., Pinty, B., and Verstraete, M. M.: Coupled surfaceatmosphere reflectance (csar) model: 2. semiempirical surface 
model usable with noaa advanced very high resolution radiometer data, J. Geophys. Res.-Atmos., 98, 20791-20801, 1993.

Schepers, D., aan de Brugh, J., Hahne, P., Butz, A., Hasekamp, O., and Landgraf, J.: Lintran v2.0: A linearised vector radiative transfer model for efficient simulation of satellite-born nadirviewing reflection measurements of cloudy atmospheres, J. Quant. Spectrosc. Ra., 149, 347-359, 2014.

Smith, L. I.: A Tutorial on Principal Components Analysis, Cornell University, USA, 51, 52, 2002.

Toon, O.: An overview of the'studies of emissions and atmospheric composition, clouds and climate coupling by regional surveys'(seac4rs) field mission, AGU Fall Meeting Abstracts, Vol. 1, 01, 2013.
Waquet, F., Cairns, B., Knobelspiesse, K., Chowdhary, J., Travis, L. D., Schmid, B., and Mishchenko, M.: Polarimetric remote sensing of aerosols over land, J. Geophys. Res.-Atmos., 114, D01206, doi:10.1029/2008JD010619, 2009a.

Waquet, F., Riedi, J., Labonnote, L., Goloub, P., Cairns, B., Deuzé, J. and Tanré, D.: Aerosol remote sensing over clouds using a-train observations, J. Atmos. Sci., 66, 2468-2480, 2009b. 